Article Unique ID: eae110

\title{
Chapter 2.3.11
}

\section{Liquid Propulsion: Propellant Feed System Design}

\section{James L. Cannon ${ }^{1}$}

Propulsion Systems Department, NASA George C. Marshall Space Flight Center, Huntsville, AL, USA

\footnotetext{
${ }^{1}$ Corresponding Contributor:

James L. Cannon

NASA George C. Marshall Space Flight Center

Mail Stop: ER01

Huntsville, AL

USA

256-544-7072 (Phone)

james.1.cannon@nasa.gov

File Name: eae110.doc (MS Word)
} 


\section{KEYWORDS}

Liquid propulsion, liquid rocket engine, engine components, propellant feed system, pump fed, pressure fed, liquid propellants, tanks, pressurant systems, feed lines, valves, turbopump, pumps, turbines, inducer, impeller, rotor, nozzle, bearings, seals.

\section{NOMENCLATURE}
$\mathrm{A}=$ area
$\mathrm{C}_{\mathrm{o}} \quad=\quad$ fluid ideal velocity
$\mathrm{C}_{\mathrm{p}}=$ specific heat
$\mathrm{D} \quad=\quad$ bore diameter, $\mathrm{mm}$
$\mathrm{g}=$ gravitational constant
$\mathrm{H}=$ pump head rise
$\mathrm{J}=$ conversion
$\mathrm{LH}_{2}=$ liquid hydrogen
LOX $=\quad$ liquid oxygen
$\mathrm{N}=$ pump rotational speed, rpm
$\mathrm{n}=$ number of stages
$\mathrm{N}_{\mathrm{s}} \quad=\quad$ specific speed
NPSH $=\quad$ net positive suction head
$\mathrm{Q}=$ volumetric flow rate
$\mathrm{P} \quad=\quad$ pressure
$\mathrm{P}_{\mathrm{o}} \quad=\quad$ inlet total pressure
$\mathrm{P}_{\mathrm{v}} \quad=\quad$ vapor pressure of fluid 


$$
\begin{array}{lll}
\mathrm{PR} & = & \text { turbine pressure ratio } \\
\mathrm{T}_{\mathrm{i}} & = & \text { inlet total temperature } \\
\mathrm{U} & = & \text { tangential blade velocity } \\
\mathrm{V} & = & \text { velocity } \\
\mathrm{I}_{\mathrm{sp}} & = & \text { specific impulse }
\end{array}
$$

Greek Symbol

$$
\begin{array}{lll}
\gamma & = & \text { specific heat ratio } \\
\rho & = & \text { fluid density }
\end{array}
$$

\begin{abstract}
The propellant feed system of a liquid rocket engine determines how the propellants are delivered from the tanks to the thrust chamber. They are generally classified as either pressure fed or pump fed. The pressure-fed system is simple and relies on tank pressures to feed the propellants into the thrust chamber. This type of system is typically used for space propulsion applications and auxiliary propulsion applications requiring low system pressures and small quantities of propellants. In contrast, the pump-fed system is used for high pressure, high performance applications. The selection of one propellant feed system over another is determined based on design trade studies at both the engine and vehicle levels. This chapter first provides a brief overview of the basic configurations of pressure-fed systems. Pump-fed systems are then discussed with greater detail given to the turbomachinery design. Selected design requirements and configurations are provided.
\end{abstract}




\section{INTRODUCTION}

The propellant feed system is a major component of a liquid rocket engine responsible for delivering the propellants from the tanks to the thrust chamber at the required flow rate and pressure conditions. The propellant feed system comprises all engine elements except the thrust chamber assembly and control system. Broadly, it consists of propellant tanks, feed lines (e.g. tubes and ducting), valves, and pressurization devices. Depending on how the propellants are pressurized and fed into the thrust chamber, the feed system is classified as pressure-fed or pump-fed as shown in Figure 1. The pressure-fed system is simple and relies on tank pressures to feed the propellants into the thrust chamber. These systems are primarily used for space propulsion applications and auxiliary propulsion applications, requiring low system pressures and smaller quantities of propellants. For high-pressure, high-performance applications, the pump-fed system is generally used by employing a turbopump. The word turbopump is used to describe rotating turbomachinery pumping the propellants.

The selection of one propellant feed system over another is determined based on design trade studies at both the engine and vehicle levels. Like any complex system, the vehicle and engine requirements must be iterated such that the engine can support the vehicle requirements. The advantages and disadvantages of pressure-fed versus pump-fed systems are traded early in the design phase. Pressure-fed systems are generally limited to relatively low chamber pressures because high pressures make the vehicle tanks too heavy. However, they can be reliable due to its reduced part count and complexity. On the other hand, the advantages of using pump-fed systems become apparent when the mission requirements dictate higher insertion velocities. The use of turbopumps enables an engine operating at high chamber pressures (i.e. high thrusts) without increasing the vehicle tank weight. The weight savings come about as a result of a 
reduction in pressure requirements on the supply tanks and their feed lines. The tank pressure becomes practically independent of the chamber pressure using the turbopump. The turbopump requirements are established by the engine power cycle being considered. Descriptions of various engine power cycles are provided in Chapter 2.3.8 (see eae107). Details about the thrust chamber design can be found in Chapter 2.3.10 (see 109).

This chapter is organized as follows. A brief overview of the basic configurations of pressure-fed systems is first given. Pump-fed systems are then discussed with greater detail given to the turbopump design. Selected design requirements and configurations are provided. Additional details about propellant feed system design are available in articles listed in the References section.

\section{PRESSURE-FED SYSTEMS}

The pressure-fed system relies on tank pressures for pressurizing the propellants. The pressurefed system generally includes: 1) pressurized tank(s) to store propellants, 2) pressurant gas or other expulsion device to provide energy for the feed system, 3) valves to control the pressure and flow, 4) ducting or piping to transfer fluids, and 5) one or more thrust chambers to generate thrust. The pressured-fed systems are classified according to the pressurant source, which determines how the propellant is expelled from the tank (Huzel and Huang, 1992). It can be as simple as a cold gas thruster, which has a pressurized tank connected to a propellant tank. Figure 2 shows two other common configurations: 1) monopropellant and 2) bipropellant systems. The monopropellant system uses a single propellant (e.g. hydrazine) flowing through a catalyst-bed

prior to expanding in a converging-diverging nozzle as shown in Figure 2(a). The pressure fed can be a little more complicated with bi-propellant systems, which employ an oxidizer tank and a 
fuel tank, both of which require a pressurant system to expel the propellant into the feed system. Figure 2(b) shows a schematic diagram of a typical bi-propellant system. Stored-gas pressurant systems are widely used. The gas is stored in a pressurant tank at pressures up to $270 \mathrm{~atm}$, supplied to the propellant tanks at a regulated pressure. Helium is commonly used due to its low molecular weight, minimizing total pressurant weight. Nonetheless, when selecting a pressurization gas, various design factors must be taken account, including mission requirements, reliability, cost, weight, size, and pressurant gas compatibility with tank materials.

The pressure-fed system has general characteristics of being a simple, low-cost design, which provides low to moderate engine performance. The system can be reliable with few parts, but are typically heavy because of the pressurized tanks. These systems are primarily used for orbit maneuver, orbit insertion, attitude control, reaction control, and small upper stage propulsion. There are operational factors influencing pressurant requirements. They include propellant vaporization, tank wall temperature, vapor condensation, solubility of the pressurization gas, ullage-gas compression, chemical reaction, and pressure-gas turbulence (Huzel and Huang, 1992).

\section{PUMP-FED SYSTEMS}

The pump-fed system uses a turbopump to pressurize and feed the propellants into the thrust chamber at relatively high pressures. The turbopump typically consists of one or more pumping elements driven by a turbine. The energy to power the turbine itself is provided by the expansion of high pressure gases, which are usually mixtures of the propellants being pumped. Depending on how these gases are generated, the system can be classified as: 1) gas generator cycle, 2) staged combustion cycle, or 3) expander cycle (see eae107). 


\subsection{Turbopump Assembly}

Figure 3 shows a cross-sectional diagram of the basic turbopump assembly of a liquid rocket engine, revealing the complicated nature of such a system. The turbopump assembly for a liquid rocket engine is a complete system in itself. It consists of many elements, some of which are themselves subsystems (e.g., pump and turbine). The turbopump consists of seven basic elements: 1) pump, 2) turbine, 3) bearings, 4) seals, 5) housings, 6) thrust piston, and 7) spline/couplings (Anon., 1974a). These basic elements along with a description of their function are schematically illustrated in Figure 3.

The turbopump is designed to maximize the pump speed and to push the turbine gas temperatures as high as possible. The turbopump speed is maximized to save weight while maintaining operational efficiency. The temperature differentials between the turbine and pump elements are substantial especially when pumping cryogenic propellants, such as liquid oxygen (LOX) and liquid hydrogen (LH2). In addition, the turbopump operates over a wide pressure range while pumping a fluid from a low pressure at the inlet to a very high pressure at the discharge. The inlet pressures to the pumps are relatively low due to low tank pressures. Thus, the potential for pump cavitation must be taken into account in the design. The cavitation is a phenomenon that occurs when the static pressure at any point in a fluid flow passage becomes less than the fluid's vapor pressure. The drive gases used to power the turbine are either highpressure combustion gases or heated gaseous fuel. The heat capacity and available energy of these combustion products is roughly ten times that of the typical jet engine. More detailed descriptions of auxiliary combustion devices can be found in Chapter 2.3.10 (see eae109). Because of the extreme turbopump environments, the bearings must be cooled and lubricated by the propellants being pumped. One critical area, which may not receive as much attention as 
other areas, is the static and dynamic seals. These elements are used to preclude the mixing of propellants within the turbopump, which can potentially result in burning or explosion if a catastrophic failure occurs.

The turbopump being a highly integrated system requires multidisciplinary engineering and coordination as well as a comprehensive set of design tools. Such complex mechanical designs require an extensive amount of engineering effort across various technical disciplines including hydrodynamics, aerodynamics, mechanical, structures, structural dynamics, rotordynamics, thermal, materials, manufacturing, testing, and instrumentation. An illustration of the multidisciplinary interaction can be found in the Figure 4, which shows a typical integrated design process. A turbopump design process can be laid out in a systematic manner starting with the establishment of turbomachinery design goals and requirements. These are derived from the engine system requirements and include propellant flow rates, propellant properties, pump outlet pressure (or discharge pressure), and turbine drive arrangement. If engines use fuel and oxidizer with similar densities, such as LOX and RP-1, the fuel and oxidizer pumps can be mounted on the same shaft and driven by a single turbine. When propellants with dissimilar densities such as LOX and LH2 are used, they require separate pumps because the head rise is much higher for the lower-density propellant. Thus, the LH2 turbopump usually requires multiple stages and will run much higher speeds than the LOX turbopump.

A general overview of the major turbopump elements are provided in the following subsections along with selected design configurations.

\subsection{Pump Elements}

The basic operational principle for a pump is to add energy to a fluid by a transfer of angular momentum between the fluid and rotating element. The changes in angular momentum 
require changes in tangential velocity. The pump consists of elements that are both stationary and rotating as shown in Figure 5. The rotating blades on an impeller or an inducer increase the fluid tangential velocity while the stationary blades decrease the fluid tangential velocity. Two types of energy conversions explain the complete pump flow process: 1) diffusion process where the kinetic energy is converted to the pressure, and 2) shaft power produces the kinetic energy, which is converted to the potential energy. The fluid enters the pump at a low pressure from the supply tank. A tank pressurization system is necessary to maintain the required net positive suction head (NPSH) to prevent pump cavitation. The pump inlet pressure is usually minimized to reduce the tank size and weight. Once the fluid enters the pump at the inducer inlet, the inducer adds energy to the fluid before passing to the impeller. The inducer needs to add sufficient energy to the fluid to suppress cavitation of the fluid passing through the impeller. It should be noted that not all designs require an inducer. The impeller adds significantly more energy in the form of kinetic energy prior to directing the flow into the diffuser and the volute.

Standard pump requirements include the inlet temperature, inlet pressure, mass flow rate, pump head rise, and NPSH. Once the pump requirements have been derived from the integrated engine level, the pump design and sizing analysis are initiated to determine the required number of pump stages, pump speed, impeller diameter, materials, tip speeds, pumping system efficiency, and horsepower. At this design stage, it will also be determined if an inducer is required.

Engine inlet conditions to the turbopump, expressed as NPSH, determine the pump suction performance requirement and is written as:

$$
N P S H=\frac{p_{o}-p_{v}}{\rho}
$$


where $P_{i}$ is the inlet total pressure, $P_{0}$ is the vapor pressure of fluid, $g_{0}$ is the gravitational constant, and $\rho$ is the fluid density. The suction performance requirement is the ability of the pump to operate at the available NPSH without experiencing severe cavitation. The pump suction performance is dependent on the pump inlet pressure, which is driven by the tank and feed system requirements. Another important pump design parameter based on pump affinity laws is the specific speed $\mathrm{N}_{\mathrm{s}}$, which establishes the relationship between the shaft speed, volumetric flow rate, and pump head and is expressed as:

$$
N_{s}=\frac{N \sqrt{Q}}{\left(\frac{H}{n}\right)^{0.75}}
$$

where $\mathrm{N}$ is the pump rotational speed, $\mathrm{Q}$ is the volumetric flow rate, $\mathrm{H}$ is the pump head rise, and $\mathrm{n}$ is the number of stages. Figure 6 shows the relationship of the pump efficiency as a function of stage specific speed for various types of pumps. The pumps are generally classified as axial flow, mixed flow, or centrifugal pumps. Among them, the centrifugal pump is typically considered the preferred choice for large liquid rocket engines (Anon., 1973). The centrifugal pumps have higher efficiencies relative to other pump types (Humble, Henry, and Larson, 1995). As shown in Figure 6, a centrifugal pump stage operates at a higher specific speed compared to most other pumps. This is due to the fact that for a given volume flow rate and head rise, the centrifugal pumps operate at higher rotational speeds, which result in smaller pump diameters and masses. From an engine operational perspective, they also have excellent off-design operating characteristics compared to axial pumps and thus greatly simplify engine startup and shutdown operational requirements. 
The centrifugal impellers can be either unshrouded or shrouded. The shrouded (or covered) impeller is preferred because you can maintain a tighter clearance, which lowers leakage and aides in thrust balance control. The shroud, however, adds mass, and thus puts higher stresses on the part. The centrifugal impeller tip speeds are limited by hardware design and material strength (e.g. $610 \mathrm{~m} / \mathrm{sec}$ with titanium for LH2 and $274 \mathrm{~m} / \mathrm{sec}$ with Inconel 718 in LOX) (Humble, Henry and Larson, 1995). The maximum head produced by a single stage is limited by the maximum allowable tip speeds. Thus, low-density fluids such as LH2 require the use of multiple stages for high-pressure applications.

The inducer is a special pump impeller usually on the same shaft and rotating at the same speed as the main impeller. It has a low head rise and therefore a relatively high specific speed. The inducer is located immediately upstream of the main impeller. It is basically an axial flow pump with a spiral impeller, and many operate under slightly cavitating conditions. Depending on the engine system, a separate boost pump may be used. The inducer stage's head rise has to be sufficiently large enough to suppress cavitation in the main pump impeller and is typically 2 $10 \%$ of the total pump head. This allows for a smaller, lighter, higher-speed main pump (Sutton and Biblarz, 2001). Similar to the impeller, the inducer blade tip speeds are also limited by hardware design and material strength (e.g. approximately $167 \mathrm{~m} / \mathrm{sec}$ ) (Stangeland, 1988).

The designer also needs to be attentive to selecting proper materials to maintain the structural integrity of the inducers, impellers, and pump housing. Other critical areas involve the propellant feed line configurations such as bends, bellows, and internal duct support structures, which can perturb the flow distribution at the pump inlet. In particular, there are two typical characteristics of internal flow, which can cause problems: 1) cavitation, and 2) damaging unsteady flows and forces (Brennen, 1994). 


\subsection{Turbine Elements}

The basic operational principle for a turbine is to remove energy from a fluid by a transfer of angular momentum between the fluid and rotating element. The changes in angular momentum require changes in tangential velocity. The turbine consists of stationary and rotating elements as shown in Figure 7. The rotating blades on a turbine disk decrease the fluid tangential velocity while the stationary blades increase the fluid tangential velocity. Two types of energy conversions explain the turbine flow process: 1) expansion process where the pressure is converted to the velocity and 2) potential energy converted to the kinetic energy and thus to the shaft power.

The turbine requirements are defined by the selected engine power cycle. The engine power balance provides the turbine-drive gas type, flow rate, inlet temperature, inlet pressure, and pressure ratio across the turbine. Once the turbine requirements have been determined and the pump horsepower requirements are known, the turbine design and sizing analysis can begin. A generalized approach for turbine design can listed as follows. First, the hot gas supply properties (e.g. inlet temperature, specific heat, and specific heat ratio) are determined. Second, the isentropic spouting velocity $\mathrm{C}_{0}$ and turbine disk size/pitch velocity are calculated. Third, the type of turbine for achieving the optimal turbine efficiency is determined. Fourth, the pump requirements are balanced. Fifth, the turbine design parameters are compared with engine power balance predictions. The turbine design process is a highly iterative process in order to satisfy the engine requirements as well as satisfy the turbine performance, materials, stress, and dynamic requirements. 
The turbine must supply the required power to drive the pump. The energy to drive the turbine is derived from the expansion of the working fluid. The fluid enters the turbine at high pressure and temperature conditions and is discharged from the turbine at a reduced pressure and temperature condition. The turbine power is supplied by a hot gas source such as a gas generator, preburner, or regenerative jacket, depending on the engine power cycle. The turbine efficiency is dependent on three factors: 1) energy content per pound of the drive gas, 2) ratio of the tangential blade velocity $\mathrm{U}$ to the fluid ideal velocity $\mathrm{C}_{0}$, and 3 ) type of turbine. The turbine velocity ratio $\mathrm{U} / \mathrm{C}_{\mathrm{o}}$ is used to empirically characterize the range of turbine efficiency in which the turbine design operates and is expressed as:

$$
\begin{gathered}
\mathrm{U} / C_{o}=U / \sqrt{2 g J C_{P} T_{i}\left[1-\left(\frac{1}{P R}\right)^{\frac{\gamma-1}{\gamma}}\right]} \text { Singe Stage Turbine } \\
U / C_{o}=\sqrt{\sum U_{n}^{2}} / C_{o} \text { Multistage Turbine }
\end{gathered}
$$

Various types of turbines are used to provide power to the pump. The turbine flow paths can be axial or radial. An axial flow path design is typically used in liquid rocket engine turbopumps due to its high efficiency and low weight. There are two primary types of axial flow turbines: 1) impulse, and 2) reaction. They can be single staged or multiple stages. A single-stage turbine has one nozzle and one rotor while a multi-stage turbine has multiple nozzles and rotors. In the impulse turbine, all the gas is expanded across the nozzle. Impulse turbines characteristically have high pressure ratios and low flows. The impulse turbine is generally single stage but can have variations such as the velocity compounded or pressure compounded. The pressure ratio is typically as high as $8-20$ (Anon., 1974b). The reaction turbine accelerates the fluid in both the nozzle and rotor. Compared to the impulse turbine, it has a low pressure ratio and a high flow with higher efficiency, peaking at higher $\mathrm{U} / \mathrm{C}_{\mathrm{o}}$. The percent reaction for the 
stage is the ratio of the enthalpy drop in the rotor to the enthalpy drop across the stage and can vary from 25 to $50 \%$ (Anon., 1974b). Each of these turbine types mention above have different velocity and pressure distributions, which result in each turbine having different efficiency characteristic as shown in Figure 8. Ultimately, the design selection is made to maximize the turbine efficiency and minimize the weight compatible with the selected shaft speed (Stangeland, 1988).

Similar to the pump, there are some structural limits on the turbine. The tip speed structural limit is generally based on the centrifugal pull that can be carried at the base of the blade airfoil for the selected material. This is usually expressed as $\mathrm{A}_{\mathrm{a}} \mathrm{N}^{2}$ (allowable annulus area times speed squared) as a function of temperature (Stangeland, 1988). Other design limits relate to the performance and geometrical design requirements.

During turbine development, various kinds of design problems can be encountered. These problems can be placed in two general categories: 1) low cycle fatigue, and 2) high cycle fatigue. The low cycle fatigue results from extreme temperature gradients on the hardware during operation. The flow path hardware such as the inlet housing, turbine blades, and nozzles can experience low cycle fatigue cracks. Other problems encountered are associated with the high cycle fatigue. The dynamic environment of the turbine either from the aerodynamic environment or blade/vane passing frequency can tune with turbine components during operation. Blades, vanes, or nozzles are usually subject to high cycle fatigue cracking because of the severe dynamic environment. Dampers can be incorporated into turbine blades at the platform or the blade tip/shroud to address blade resonances. 


\subsection{Mechanical Elements (Bearings and Seals)}

\subsubsection{Bearings}

The turbopump shaft is supported by two or more bearings, which are designed to transmit all the fixed and dynamic loads acting on the rotor assembly to the housing (Anon., 1971). Bearings control both the radial and axial motions of the turbopump rotor. Radial control of the high-speed rotor is critical in centering the rotor in the turbopump housing to prevent rubs and maintain radial clearance to reduce parasitic flow losses. Bearing designs provide axial control of the rotor during the start and shutdown phases of the turbopump operation in response to rapid changes in pressure, temperature, and rotor speed. The bearings selected for a turbopump design must be able to handle the operational conditions. They must provide adequate radial stiffness and damping for rotordynamic control. The fluid being pumped is generally used to cool the bearings. Cryogenic fluids such as LH2 and LO2 provide little or no lubrication. RP1 , on the other hand, does provide some lubrication.

One of the design limitations of rolling element bearings is the speed. The typical parameter used to express this limitation is the DN limit. The DN is defined as the product of the inner-race bore diameter $\mathrm{D}$ (in $\mathrm{mm}$ ) and the pump rotational speed $\mathrm{N}$ (in $\mathrm{rpm}$ ). The $\mathrm{DN}$ limit ranges from 1.6 million to 2.1 million (Huzel and Huang, 1992). The shaft diameter along with splines and couplings must be properly sized to transfer torque that is a function of speed and horsepower (Stangeland, 1988). Once the shaft is sized, the bearing inner race diameter is established. There are two major bearing types used in the turbopumps: 1) rolling elements, and 2) fluid film. The rolling element bearings consist of a cage (or ball separator), inner raceway, outer raceway, and rolling element ball (or roller) as shown in Figure 9. The primary failure modes of rolling element bearings are wear and fatigue, which limits life. Rolling element 
bearings, roller bearings, and ball bearings are the most commonly used because of their capabilities in load, speed, stiffness, and misalignment tolerances. The selection of bearing materials begins with understanding their compatibility with the propellants to be used. Bearings have been typically made of $440 \mathrm{C}$ material, which is corrosion resistant steel. Since most propellants are poor lubricants, lubrication for the bearing is provided by either a baked on dry film lubrication, burnishing raceways, or transferring lubrication for the cage or bearing separator. The cage or ball separator is typically made of Armalon, which is a glass filled polytetrafluoroethylene (PFTE) material. During bearing operation, the balls contact the cage transferring a thin film of lubrication to the balls and raceways.

Another bearing type, which is gradually gaining acceptance in liquid rocket engine turbomachinery, is the fluid film bearing such as the hydrostatic bearing. Hydrostatic bearings have the potential benefit to increase bearing life and remove the speed limit associated with rolling elements. One of the dominant features of the hydrostatic bearing is the significant stiffness and damping (minimal with rolling elements), which can be obtained given sufficient pressure and flow. These features are desirable to have in terms of rotordynamic characteristics. Rotors supported by hydrostatic bearings must be controlled during transient operation such as the engine startup and shutdown to prevent rubbing. The materials selected for hydrostatic bearing application must be rubbing tolerant and fluid compatible.

\subsubsection{Dynamic Seals}

The turbopumps use dynamic shaft seals between rotating and stationary parts. The primary purposes for dynamics seals are to minimize internal parasitic flows and to separate propellants such as the oxidizer from the fuel. Seal failures can cause catastrophic turbopump failures as a result of propellant mixing internally within the turbopump as well as rubs in oxidizing 
environments. A dynamic seal system for an interpropellant seal package consists of two facecontact seals (one for the LOX side and one for the hot gas), a purged double circumferential intermediate seal, drains, and purges. The leakage from the face-contact seals is drained through separate drain systems, which need to be properly sized. The double circumferential seal is inertgas purged, usually with gaseous helium, providing effective separation of the drain cavities in the event of a seal failure. Reliable operation of the turbopump requires a seal system designed to: 1) minimize the severity of seal operating conditions, 2) provide allowances for all possible operational extremes, and 3) allow for seal failure without destructive failure of the turbopump (Anon., 1978). A good example or illustration is a seal used to separate two incompatible fluids such as LOX and RP-1 or LH2 as shown in Figure 10.

Meeting all the design requirements for dynamic seals is critical and challenging. Dynamic seals are usually exposed to fluids, which are non-lubricating. They deal with large thermal gradients and high pressures, presenting fluid compatibility issues. After considering all the mechanical, fluid, and thermal issues, one must consider the dynamics loads on the seals, which can be induced by fluids or mechanically. These dynamic loads can occur during steady state or transient operation. Dynamic seals with bellows typically require dampers to address structural dynamic concerns.

The primary types of rotating seals used in the turbopumps are the face-contact and circumferential seals. Face-contact seals use face seals in rubbing contact with a mating ring for sealing the fluid. Circumferential seals in interpropellant seals are used for sealing turbine hot gases. Circumferential seal designs can be either segmented seals/floating ring rubbing-contact seals or clearance gap type labyrinth seals. As with other elements in the turbopump design, rubbing contact seals are surface speed limited due to the heat generated by friction torque at the 
seal interface and the capacity of the interface to remove heat. A design guide for establishing a face load and surface speed limits was developed by studying the heat generation and speed limit as a function of surface temperature. The pressure $\times$ velocity $(\mathrm{PV})$ is proportional to the heat generation per unit area and is a better indication of heat-transfer capacity (Anon., 1978). The PV factor limits for face contact seals in various fluids have been developed and are used during the seal design process. A detail structural, thermal, and flow model of the seal system is needed to ensure all seal fits, clearances, leakage flows, thermal limits on materials are maintained during all phases of operation.

\subsection{Rotordynamics}

Rotordynamics is a specialized area of structural dynamics, which deals with the vibration of the rotating assembly and interaction with the stationary hardware. Modern turbomachinery systems are continuing to push the speed and power density. This means the rotordynamic design tools are being pushed as well. The importance of doing a credible job in analyzing the rotor system cannot be over emphasized. Rotordynamics is the key in determining the reliability of turbomachinery. For example, when a turbopump encounters a vibration problem, it has far reaching programmatic and economic consequences such as halting the entire engine development program. This happened in 1976 to the Space Shuttle main engine program when its high-pressure fuel turbopump encountered a sub-synchronous whirl problem, which required approximately ten months to resolve.

The rotating assembly of a rocket engine turbopump consists of many parts including impellers, turbine disks, shaft, seals, spacers, and bearings. The rotor assemblies can have many resonances (or natural frequencies). When the natural frequency of the rotor system synchronizes with the frequency of the forcing function, this is called a critical speed. These natural 
frequencies can be excited by many sources, such as the shaft-rotating unbalance, blade/vane passing frequency excitation, rubbing between rotating and stationary parts, seal dynamic forces, housing stiffness asymmetry, hydrodynamic forces, and aerodynamics forces. The primary objectives of rotordynamics are to determine the natural frequencies of a rotor assembly and to design the system around them (Leader, 1984).

A number of design factors such as the bearing stiffness, bearing span, shaft diameter, mass placement, and overhung mass must be considered in rotordynamics. Among these factors, the bearing stiffness is the most important parameter. The bearings used to support a rotor can be either rolling elements or fluid film. The type of bearing selected will determine the amount of stiffness and damping available. Rolling element bearings provide very little, if any, damping but provide a fixed amount of stiffness over the speed range. Fluid film bearings, on the other hand, have operational characteristics in terms of stiffness and damping, which change as a function of speed. Damping is the only mechanism, which can be introduced into the system to control or dissipate rotor energy. To be effective, damping must be placed at a location, where experiences motion.

Other design factors such as the bearing span, shaft diameter, mass placement, and overhung mass also influence the rotor natural frequencies and mode shapes. Once the system's natural frequencies and mode shapes have been determined through analysis, one can make design changes if necessary to adjust one or more of the key design factors addressed above.

\subsection{Thrust Balance System}

It is a major challenge for a turbopump designer to manage the axial thrust forces generated by the pressure and fluid momentum changes in the pump and turbine elements. The axial bearing 
loads need be maintained within the bearings capability through all phases of operations, including startup transient, main stage, and shutdown transient. Several design methods are available to the designer to balance the axial thrust: 1) impeller balance ribs, 2) impeller seals, 3) anti-vortex ribs, 4) self-compensating balance piston, and 5) thrust bearings. These methods can used by themselves or in a combination as shown in Figure 11. Modeling of the thrust balance system to determine the unbalanced force and its direction is difficult. Thus, model uncertainties must be taken into account in predicting the operational clearances. In particular, startup and shutdown transient predictions are the most difficult, and thus generally require test data for model validation. The predicted amount of unbalanced force will help determine the bearing axial load capacity requirement. The critical flow path clearances must be held to tight tolerances to meet the performance requirements while avoiding rubbing.

\section{SUMMARY}

The propellant feed system delivers the propellants from the tanks to the thrust chamber. Depending on how the propellants are pressurized, they are classified as either pressure fed or pump fed. The pressure-fed system is simple, relying on tank pressures to feed the propellants into the thrust chamber. This system is limited to relatively low chamber pressures because higher pressures make the propellant tanks too heavy. On the other hand, the pump-fed system enables high chamber pressures independent of the tanks using a turbopump. The turbopump is a highly complex, integrated system requiring multidisciplinary engineering and coordination. The selection of one propellant feed system over another must be made after design trades at the integrated system level. 


\section{ACKNOWLEDGMENTS}

I would like to extend my sincere appreciation to Eun S. Kim of The Aerospace Corporation for his help and comments in preparing this manuscript. I would like to thank David Vaughan of Jet Propulsion Laboratory for the use of his figures as well as his technical review of the article. Many thanks go to Henry Stinson, retired from NASA Marshall Space Flight Center, for his technical review and insightful feedback. Insightful suggestions and feedback were also provided by Bill Campbell, retired from Aerojet.

\section{REFERENCES}

Anon., "Liquid Rocket Engine Turbopump Bearings," NASA Space Vehicle Design Criteria Monograph, NASA SP-8048, March 1971.

Anon., "Liquid Rocket Engine Centrifugal Flow Turbopumps," NASA Space Vehicle Design Criteria Monograph, NASA SP-8109, December 1973.

Anon., "Turbopump Systems for Liquid Rocket Engines," NASA Space Vehicle Design Criteria Monograph, NASA SP-8107, August 1974.

Anon., "Liquid Rocket Engine Turbines," NASA Space Vehicle Design Criteria Monograph, NASA SP-8110, January 1974.

Anon., "Liquid Rocket Engine Turbopump Rotating Shaft Seals," NASA Space Vehicle Design Criteria Monograph, NASA SP-8121, February 1978.

Brennen, C.E., Hydrodynamics of Pumps, Oxford University Press and Concepts, ETI Inc, 1994. Humble, R.W., Henry, G.N., Larson, W.J., Space Propulsion Analysis and Design, 1st ed. Revised, McGraw-Hill, New York, 1995. 
Huzel, D.K. and Huang, D.H. Design of Liquid-Propellant Rocket Engine, revised ed., Vol 147, Progress in Astronautics and Aeronautics, AIAA, Reston, Virginia, 1992.

Leader, M. E., "Introduction to Rotordynamics of Pumps without Fluid Forces," Proceedings of the First International Pump Symposium, 1984.

Stangeland, J.L., "Turbopumps for Liquid Rocket Engines," Threshold, and Engineering Journal for Power Technology, No. 3, Rocketdyne Propulsion and Power, 1988.

Sutton, G.P. and Biblarz, O., Rocket Propulsion Elements, 7th ed., John Wiley and Sons, Hoboken, NJ, 2001.

\section{FIGURE CAPTIONS}

Figure 1 Pressure Fed and. Pump Fed Systems

Figure 2 Monopropellant System vs Bi-Propellant Liquid Propulsion System

Figure 3 Basic Turbopump Elements

Figure 4 Simplified Integrated Design Process

Figure 5 Elements of a Pump

Figure 6 Efficiency vs Stage Specific Speed for Various Types of Impellers

Figure 7 Elements of a Turbine

Figure 8 Typical Design Point Efficiencies for 1 -,2 -,3,-, and

4-Stage Turbines

Figure 9 Examples of Ball Bearing and Roller Bearing

Figure 10 Four Seal System for Separating Lox and RP-1 in F-1 Turbopump

Figure 11 Methods for Balancing Axial Thrust 
Article Unique ID - eae110

Encyclopedia of Aerospace Engineering, Volume 2 Propulsion \& Power, Wiley Publishers 


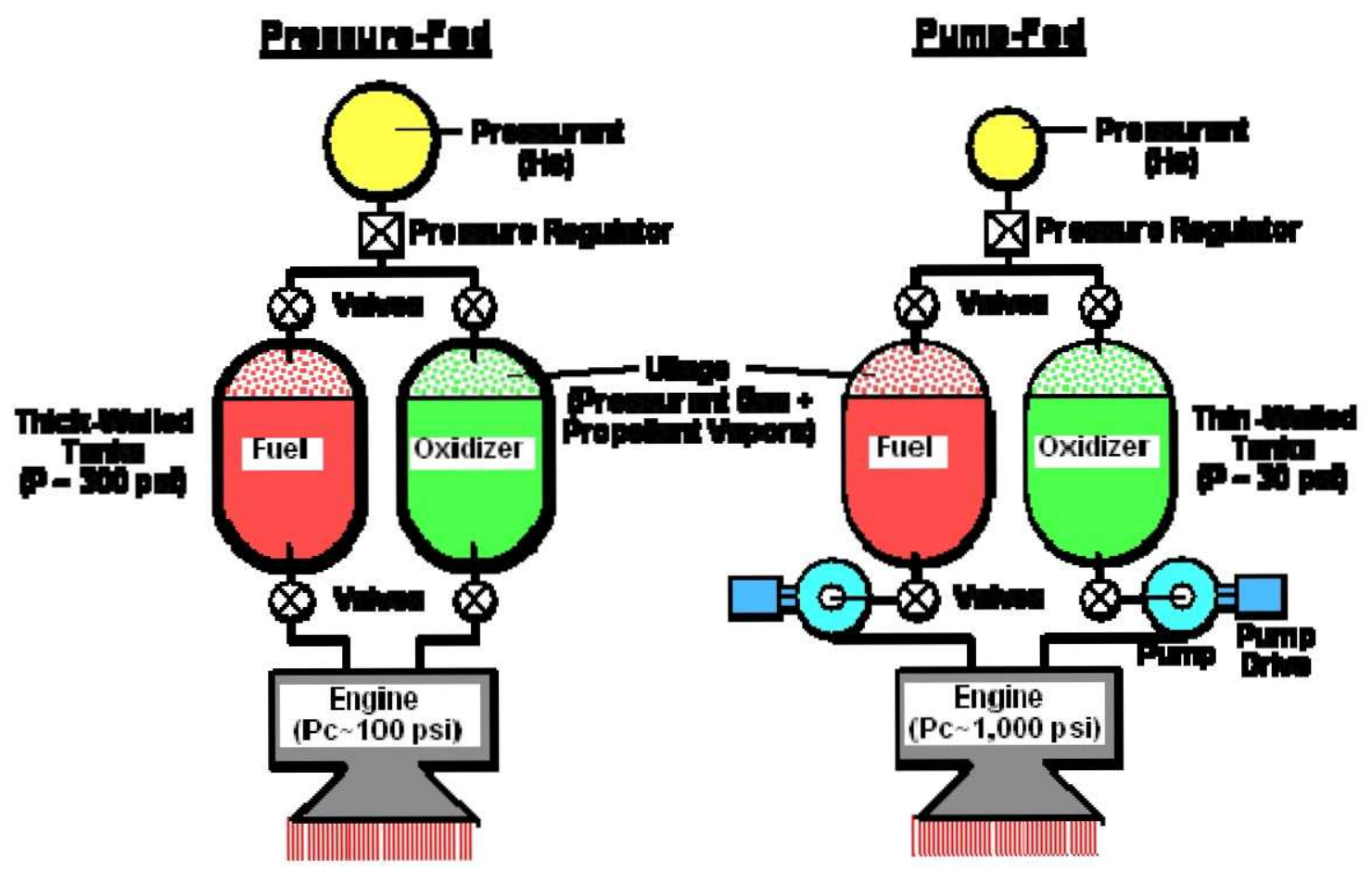

Figure 1 Pressure-Fed and Pump-Fed Liquid Propulsion 


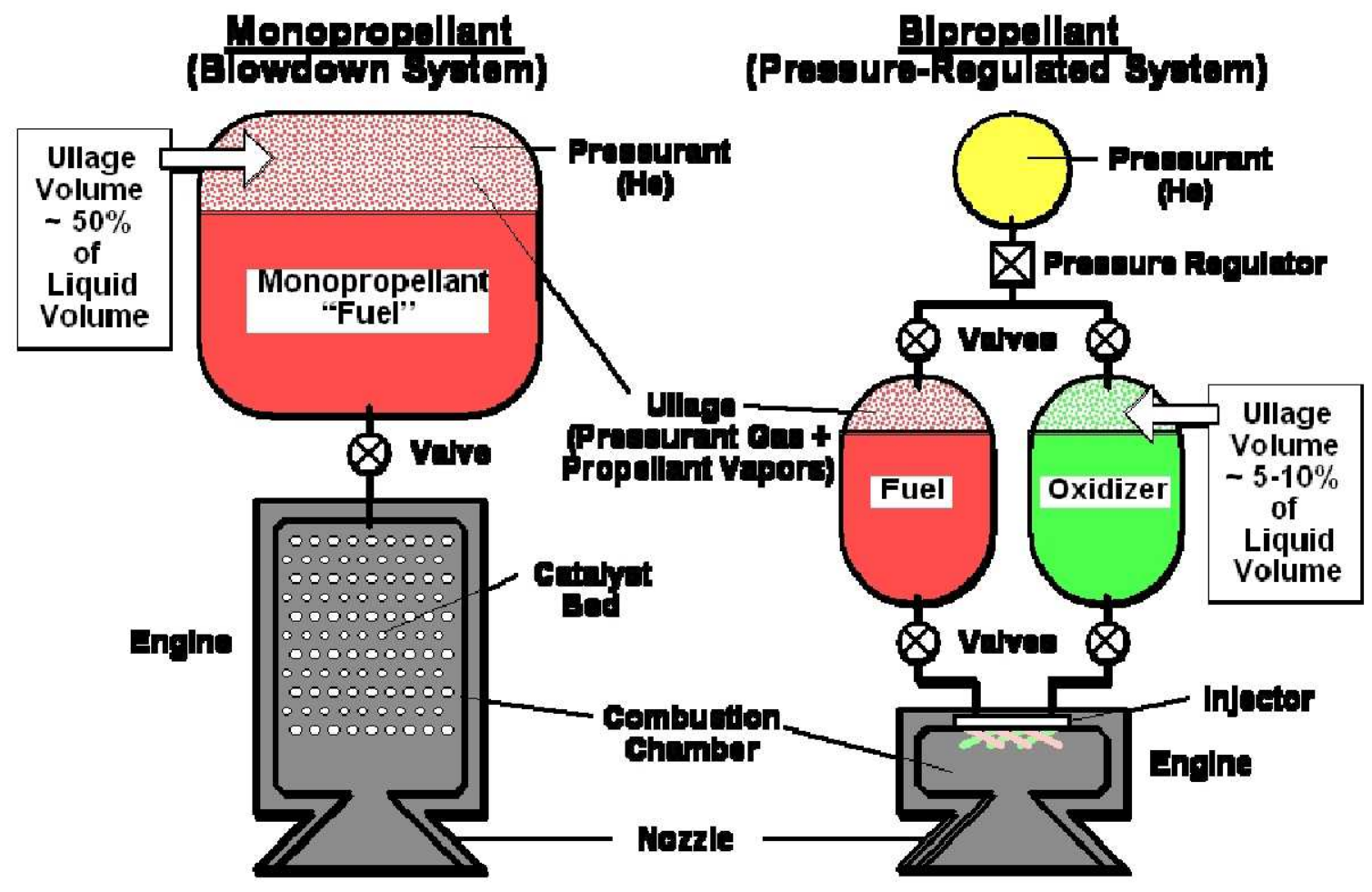

Figure 2 Monopropellant system, and Bi-propellant Liquid Propulsion System 


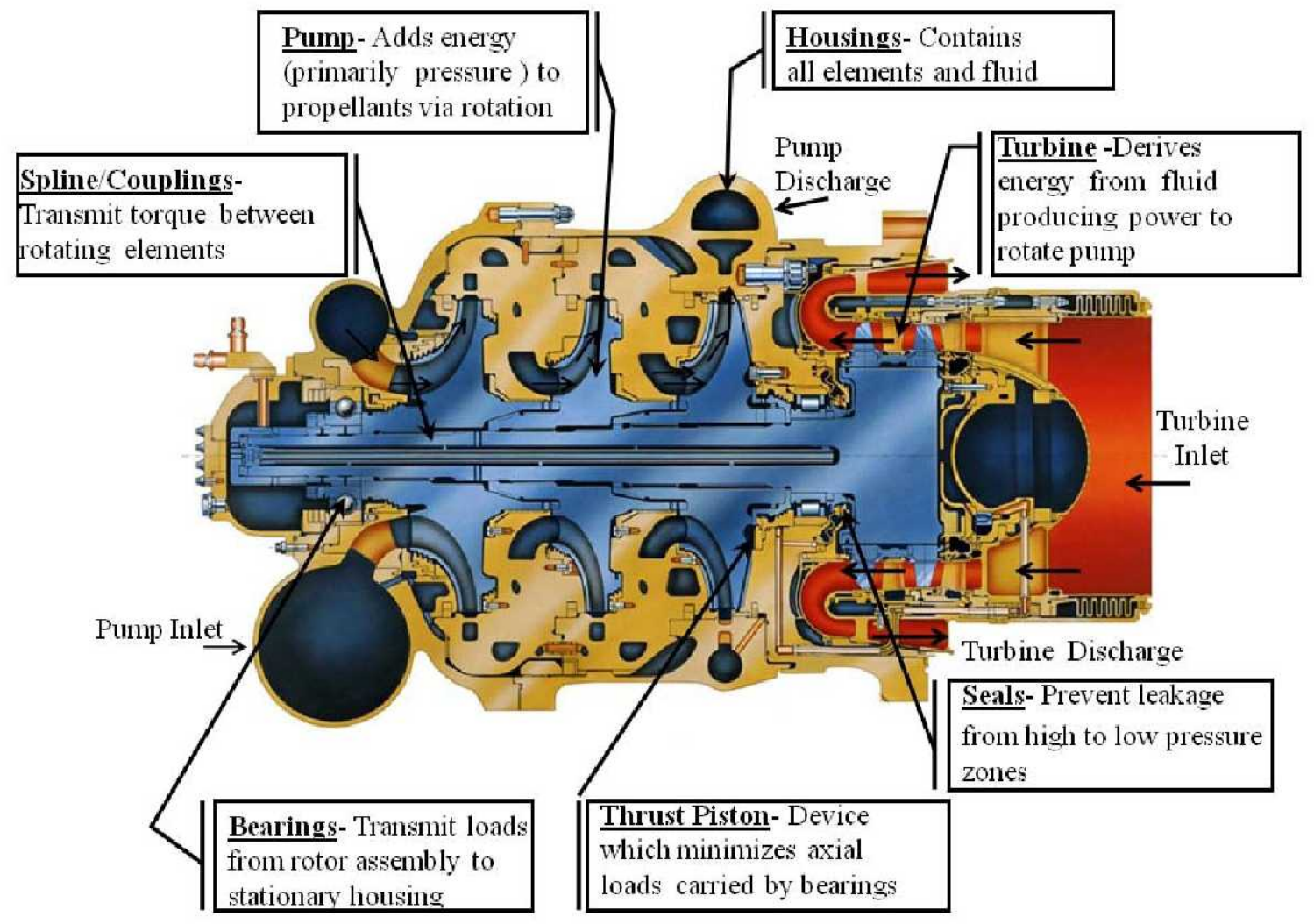

Figure 3 Basic Turbopump Elements 


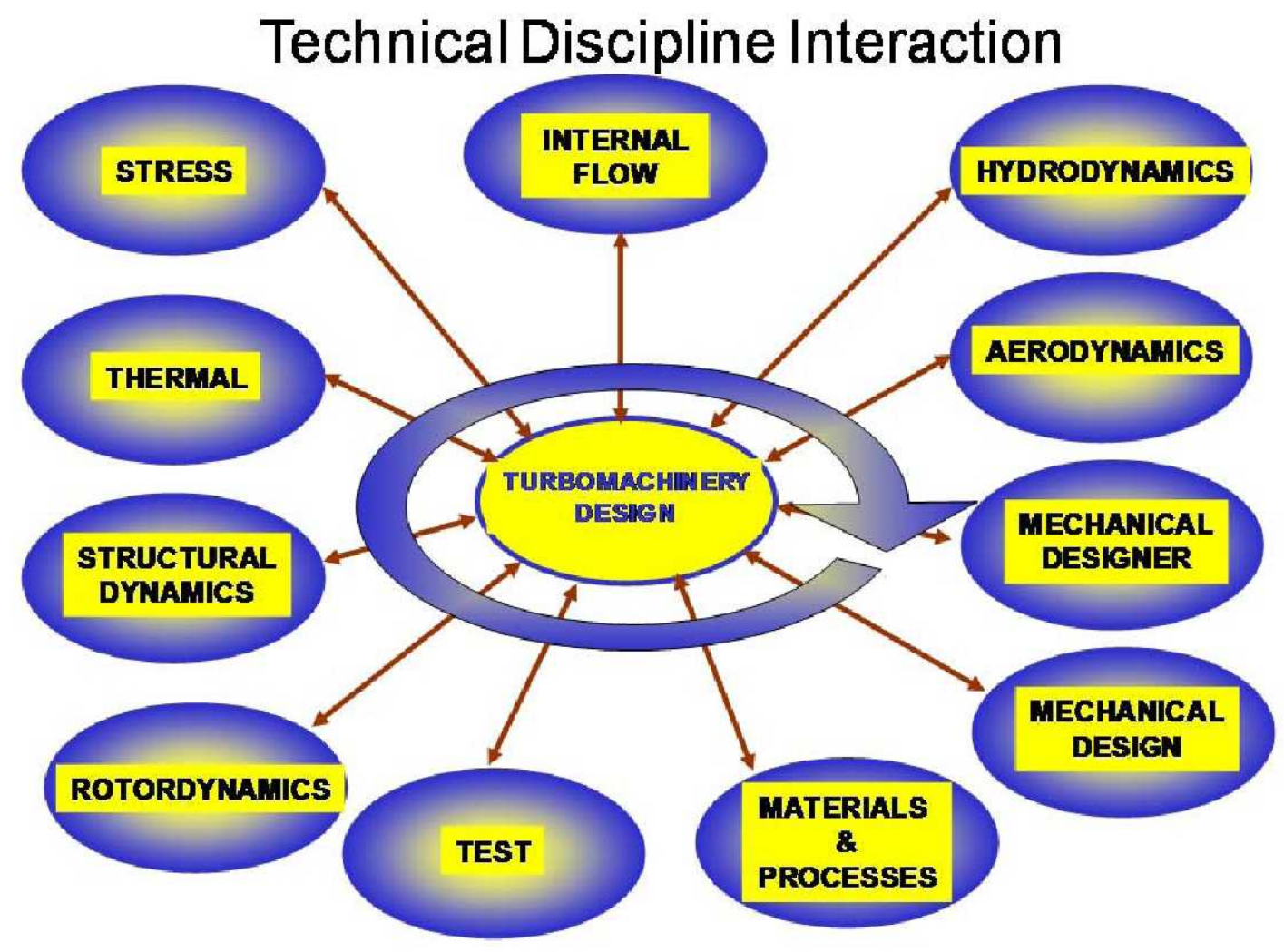

Figure 4 Simplified Integrated Design Process 


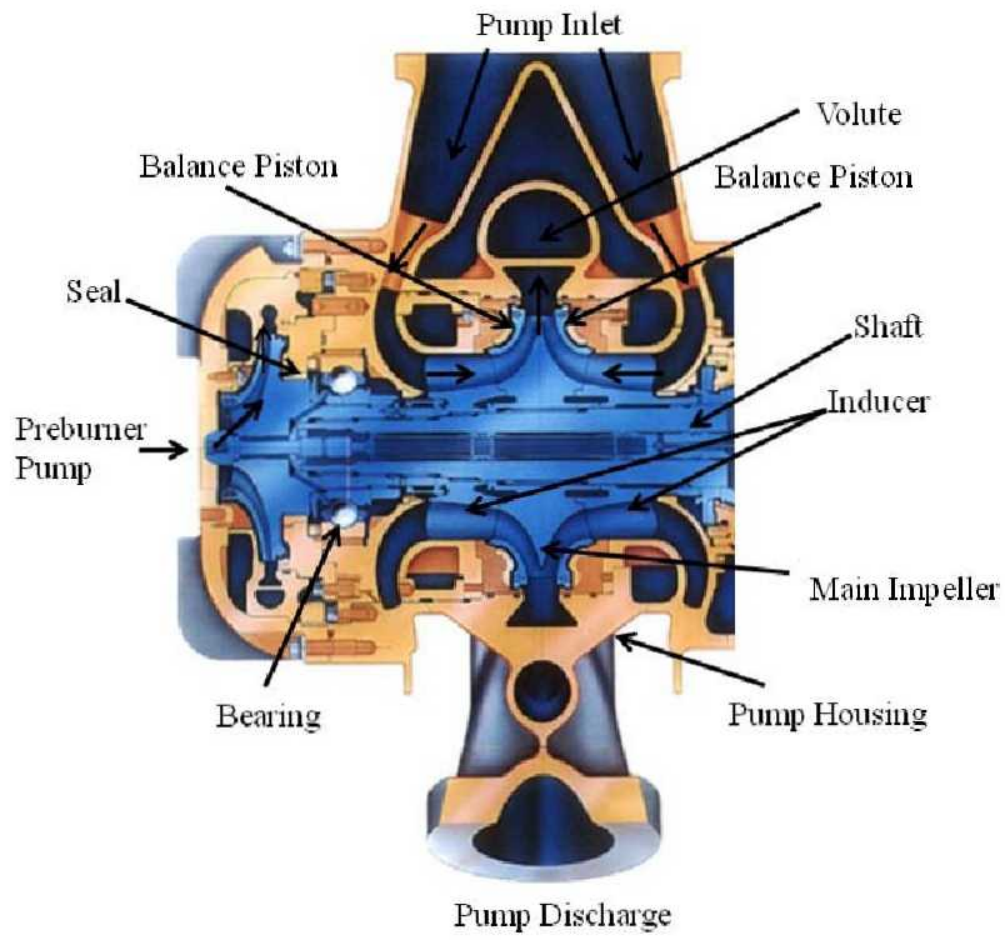

Figure 5 Elements of a Pump 


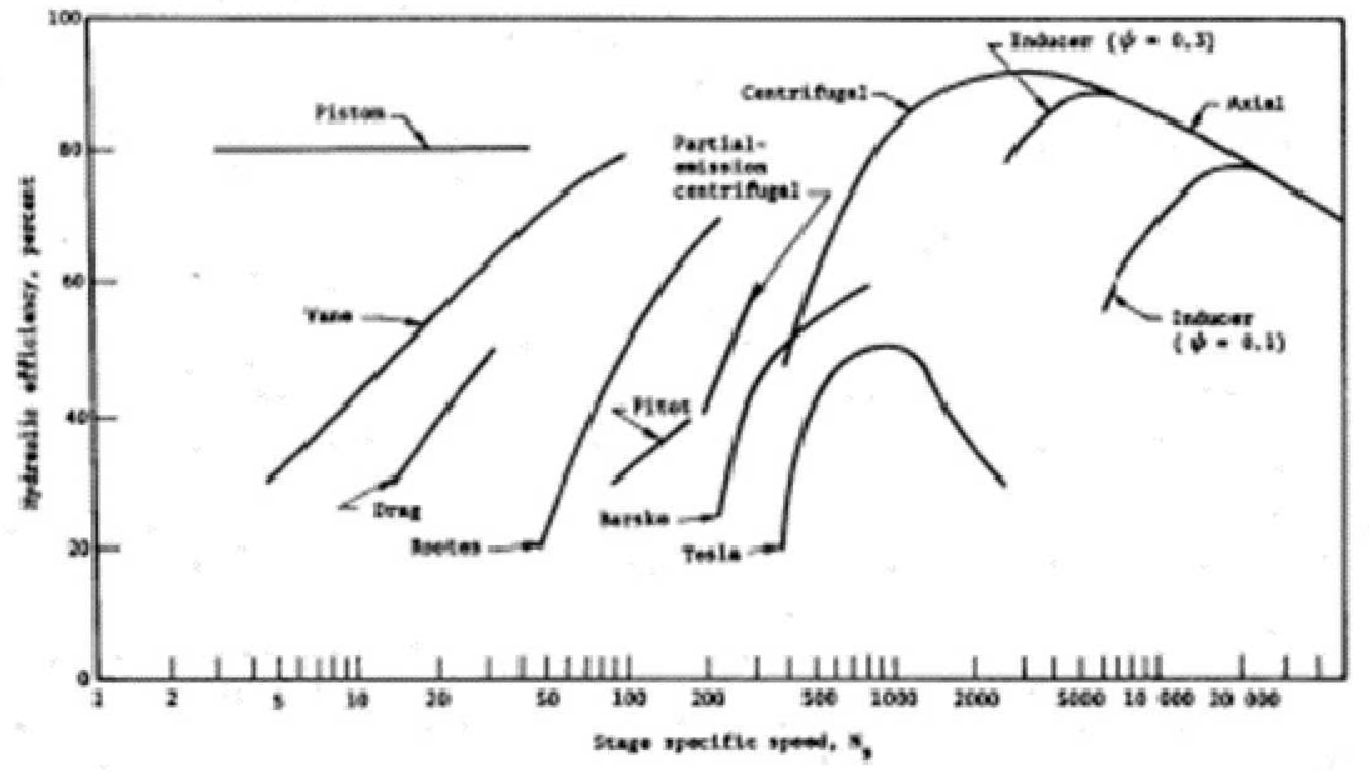

Figure 6 Efficiency vs Stage Specific Speed for Various Types of Pumps 


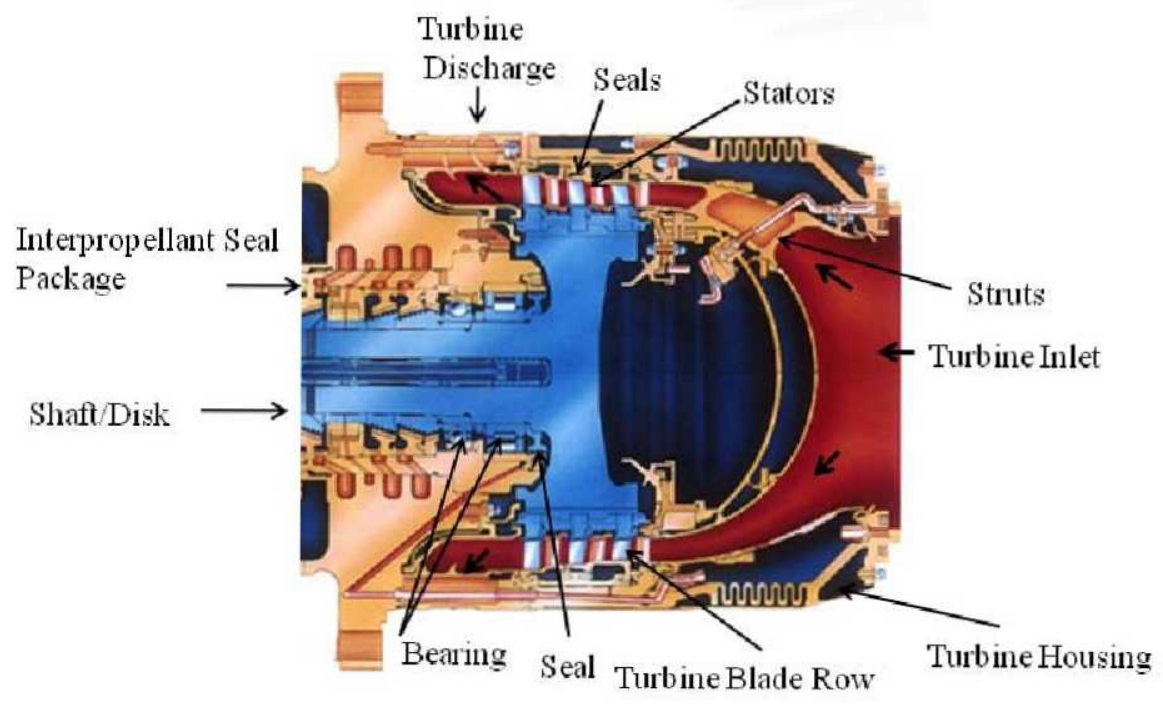

Figure 7 Elements of a Turbine 


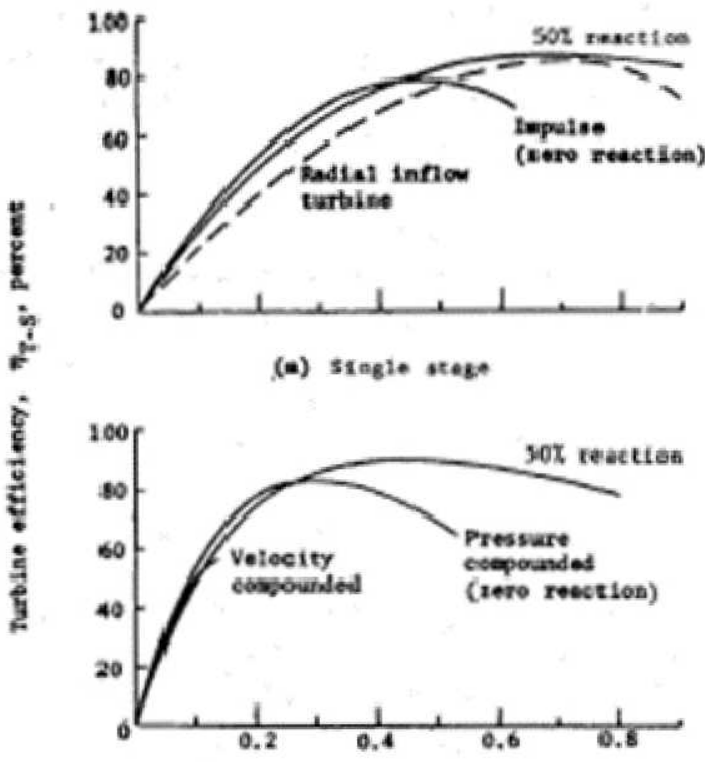

(c) Three atuge (three row for ve)

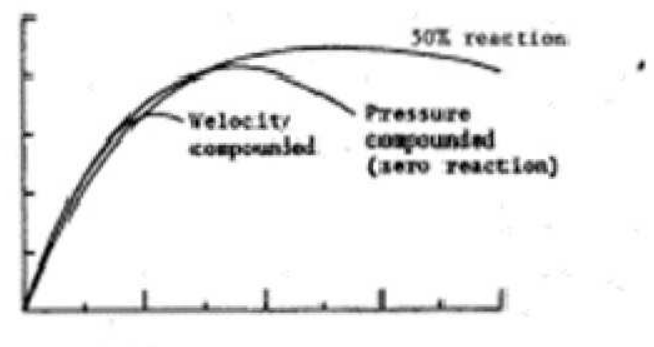

(b) Two atage (two ros tor w)

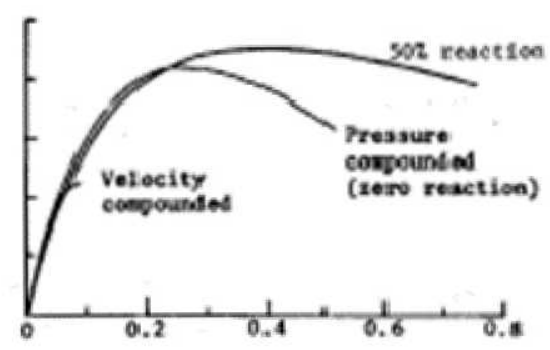

(d) Tour stage (fror now for wC)

Turbline velectity ratio, $U / C_{\text {e }}$

Figure 8 Typical Design Point $n$ for 1,2.3, and 4 Stage Turbines 


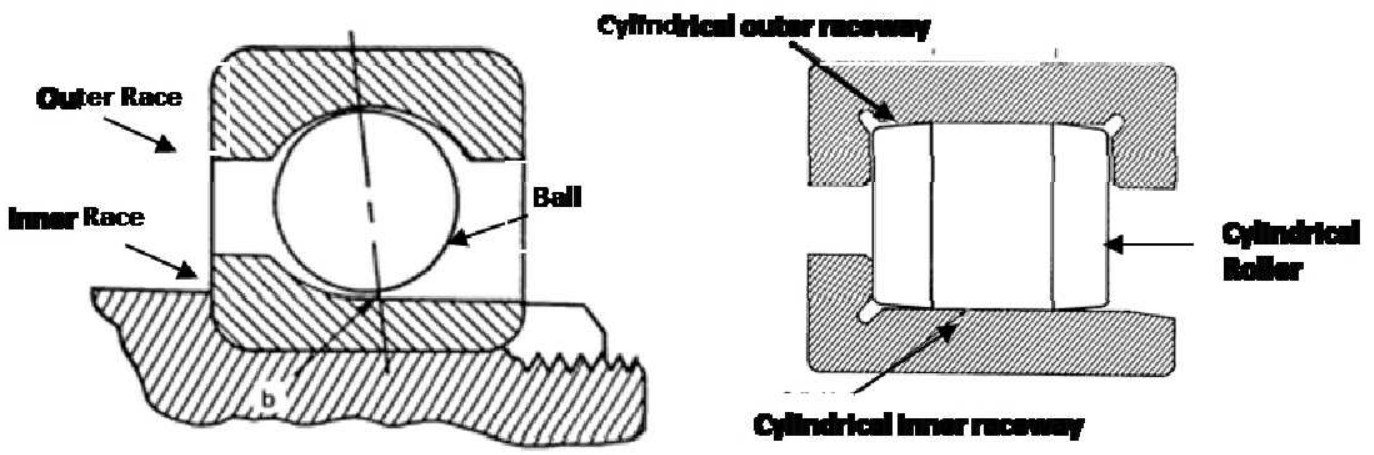

Figure 9 Examples of a Ball Bearing and Roller Bearing 


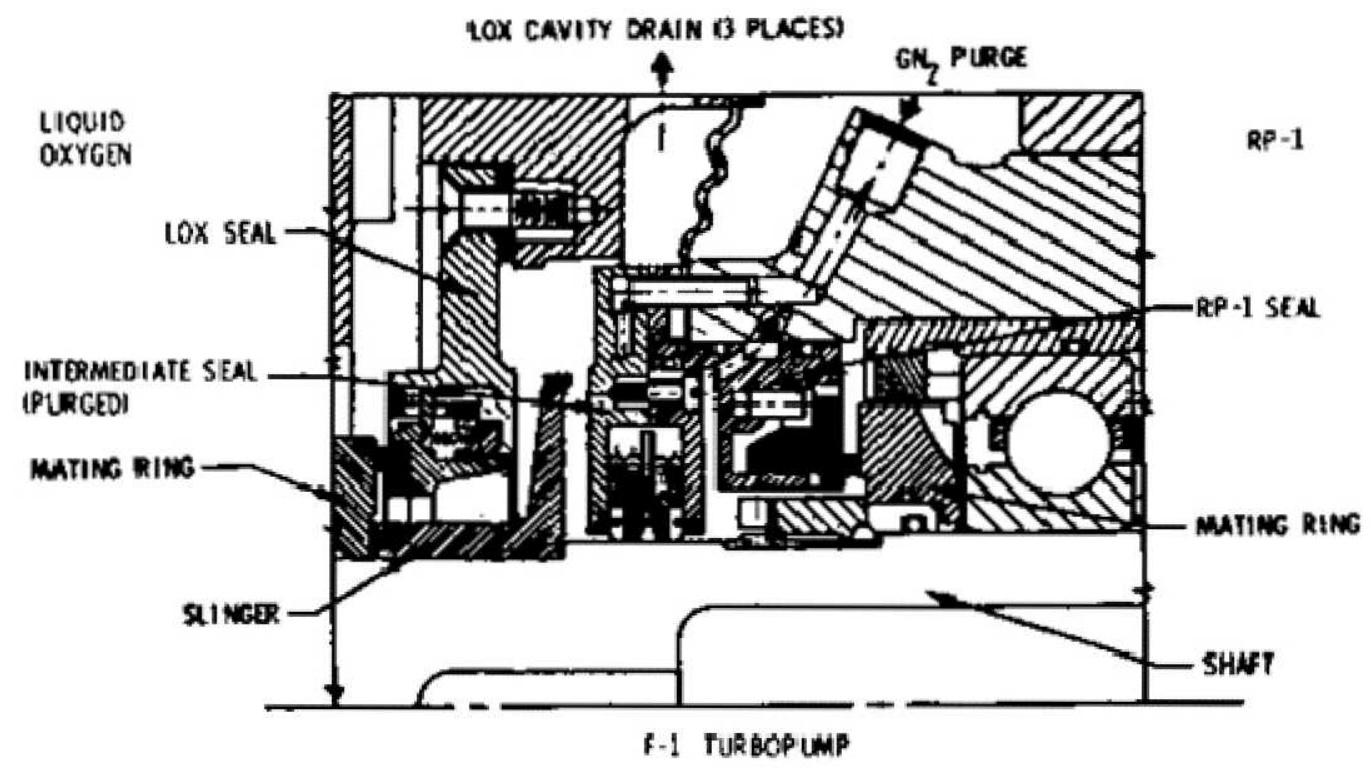

Figure 10 Four Seal System for Separating Lox and RP-1 in F-1 Turbopump 


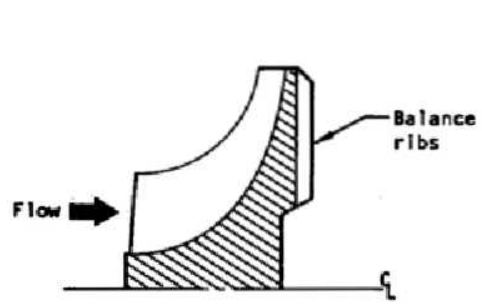

(a) Open-face Impeller with balance ribs

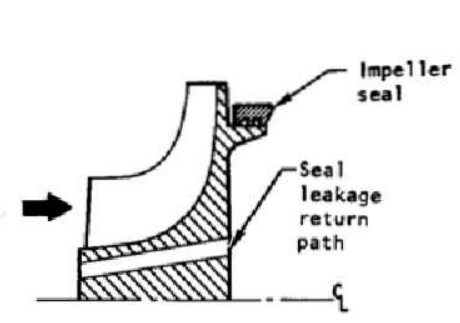

(c) Open-face impeller with hub seal

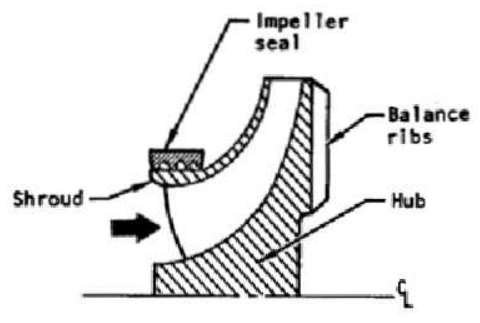

(b) Shrouded impeller with inlet seal and balance ribs

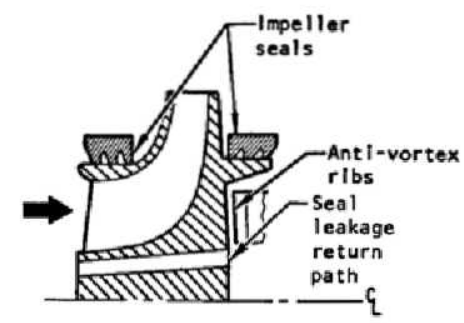

(d) Shrouded impeller with inlet and hub seals and ant $i$-vortex ribs

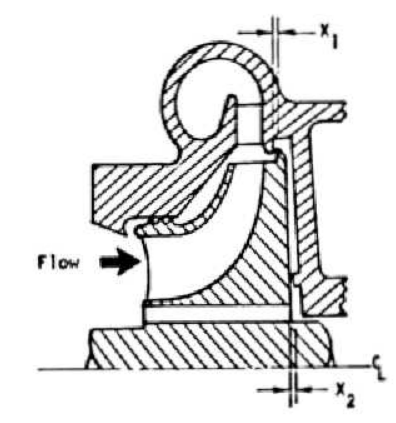

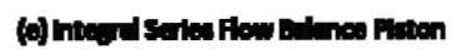

Figure 11 Methods for Balancing Axial Thrust 